\title{
Braids and Factorizable Inverse Monoids
}

\author{
David Easdown, James East and D.G. FitzGerald
}

\begin{abstract}
What is the untangling effect on a braid if one is allowed to snip a string, or if two specified strings are allowed to pass through each other, or even allowed to merge and part as newly reconstituted strings? To calculate the effects, one works in an appropriate factorizable inverse monoid, some aspects of a general theory of which are discussed in this paper. The coset monoid of a group arises, and turns out to have a universal property within a certain class of factorizable inverse monoids. This theory is dual to the classical construction of fundamental inverse semigroups from semilattices. In our braid examples, we will focus mainly on the "merge and part" alternative, and introduce a monoid which is a natural preimage of the largest factorizable inverse submonoid of the dual symmetric inverse monoid on a finite set, and prove that it embeds in the coset monoid of the braid group.
\end{abstract}

\section{Introduction}

The motivation for this work comes from several directions. In Birman's theory of knot invariants [2], the singular braid monoid plays a role, in which strings are allowed to touch, creating "double points". One can attempt to simplify a knot or link by allowing one string to pass through another. The moment at which strings touch is a "double point" and a "singular" knot or link is created. These singular versions then feature in recursive formulae for invariants such as the Alexander and Jones polynomials. Braids close up 
to form knots and links (Alexander's Theorem), so it is useful to investigate means by which braids may be simplified or modified by some manipulation of the strings. This is the idea which lead to the singular braid monoid, first introduced by Baez [1], and developed by Birman [2]. The question of what happens when we snip one or more strings has been fully investigated by Easdown and Lavers [3], leading to a preimage of the symmetric inverse monoid of a finite set, known as the inverse braid monoid, exactly analogous to the relationship between the braid group and the symmetric group.

FitGerald and Leech [5] use duality in category theory to introduce new classes of inverse monoids, a special case of which is $\mathcal{I}_{X}^{*}$, the dual symmetric inverse monoid on a set $X$, which comprises biequivalences on $X$ and a binary operation involving composition and joins of equivalence relations. One may ask (and this remains unresolved) whether $\mathcal{I}_{X}^{*}$ has a natural preimage involving braids or a modification of braids. Below we study a candidate, the merge and part braid monoid, for what might be a useful preimage of $\mathcal{F}_{X}^{*}$, the largest factorizable inverse monoid of $\mathcal{I}_{X}^{*}$. The ingredients are braids and equivalence relations on strings, where equivalent strings are allowed to touch, merge and then part as reconstituted strings. Another possibility, not dealt with here in any detail, is to allow equivalent strings just to pass through each other. This leads to the permeable braid monoid, studied in detail by East [4], who also discusses decision problems and presentations for both types of braid monoids, and explores relationships with Coxeter groups in general.

As a simple illustration of these ideas, consider the pure braid $\beta$ on 4 strings depicted in Figure 1 . Certainly $\beta$ cannot by continuously deformed into the identity braid, denoted by 1 . This follows quickly from the fact that the pure braid group is an iterated semidirect product of free groups. However, to see this directly, without the theory of pure braids, one can argue as follows. Suppose $\beta$ is equivalent to 1. If we ignore ("snip and shrivel") the second and third strings, then the configurations in Figure 2 are certainly also equivalent. But taking the projection onto a horizontal plane, the first 


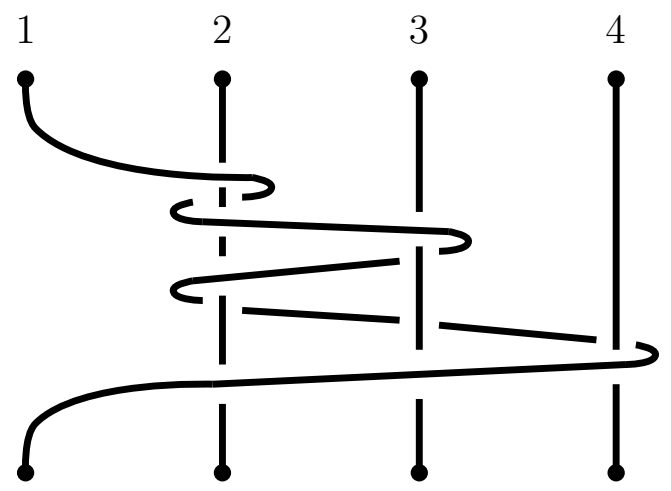

Figure 1: the pure braid $\beta$
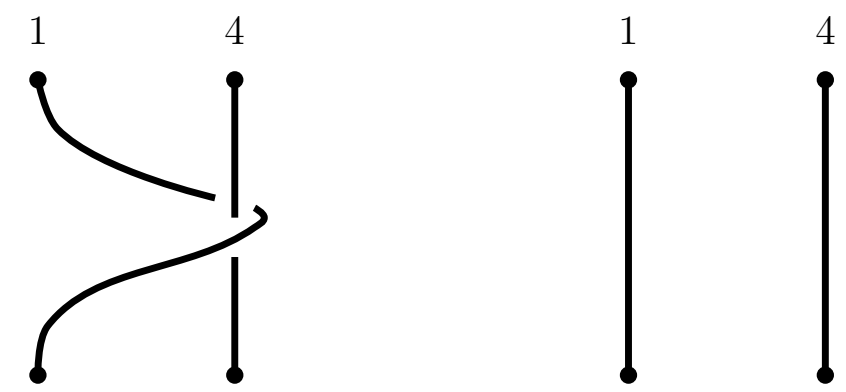

Figure 2: $\beta$ and the identity braid simplify after removing the second and third strings

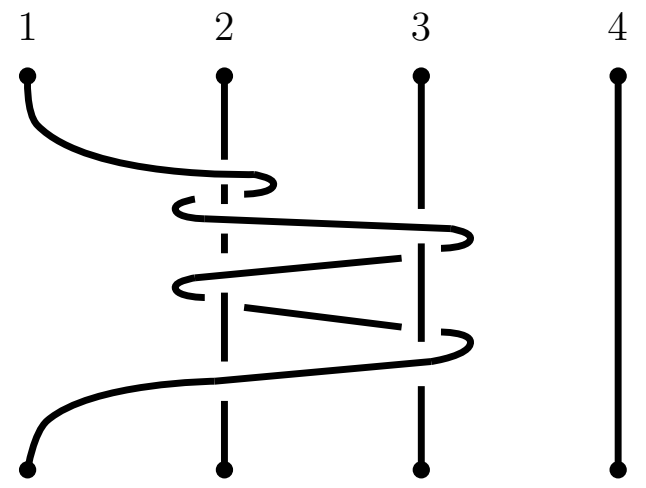

Figure 3: the pure braid $\beta^{\prime}$ 
of these produces a loop at the first point containing the fourth point, whilst the second produces a degenerate loop at the first point with the fourth point on the outside:
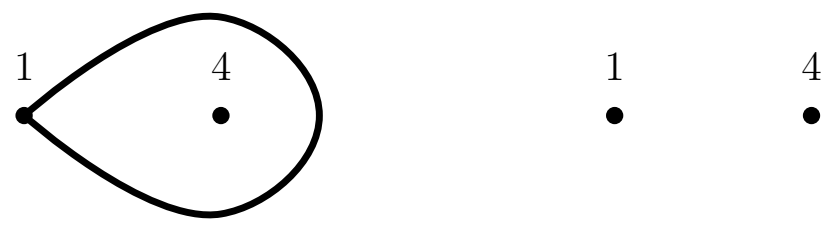

The fourth point remaining inside a loop at the first point is an invariant of a continous deformation of the first braid. This invariant fails in the horizontal projection of the final braid, which gives a contradiction.

What happens if we wish to simplify $\beta$ by allowing the first and fourth strings only to pass through each other? (This is an instance working within the permeable braid monoid studied by East [4].) The first, second and third strings are not allowed to touch, as usual, during any continuous deformation. For example, $\beta$ can become $\beta^{\prime}$ in Figure 3, which in turn can be represented by the configuration in Figure 4, where $x$ and $y$ are canonical generators of a free subgroup of the pure braid group on the first 3 strings. Hence there is no hope of transforming $\beta^{\prime}$ and hence also $\beta$ into 1 , since the commutator $x y x^{-1} y^{-1}$ is nontrivial in the free group.

What can happen if we modify the rules further, and now allow the first and fourth strings to touch, and, at the moment of touching, forget where the respective parts of strings came from, and then part as newly constituted strings? In this case, one can see using Figures 4 and 5 that $\beta$ unravels completely! In Figure 4 a dotted line indicates where the fourth string can be stretched behind other strings and made to intersect with the first string, thus "merging". Parting as newly reconstituted strings, as indicated by Figure 5, creates a configuration which can easily be seen to unwrap, so represents the identity braid. These diagrams prove that in the merge and part monoid, defined in the next section, $x y x^{-1} y^{-1}$ is trivial. This verifies one of the relations in a presentation (see [4]). 


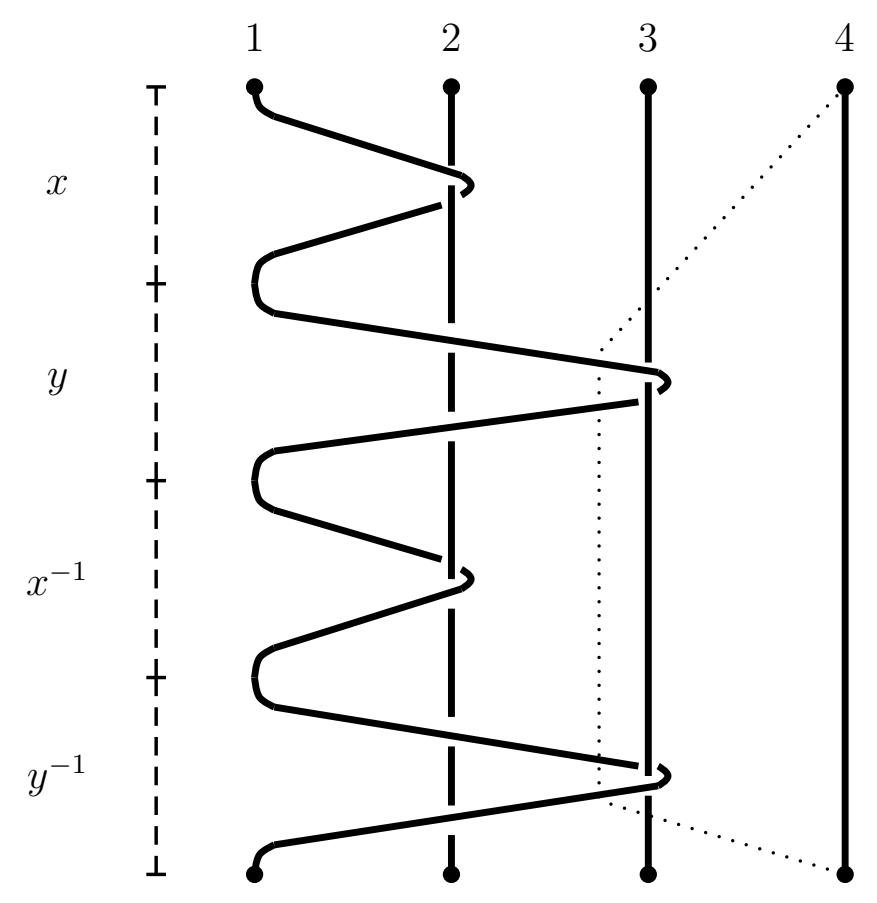

Figure 4: representing $\beta^{\prime}$ by a commutator

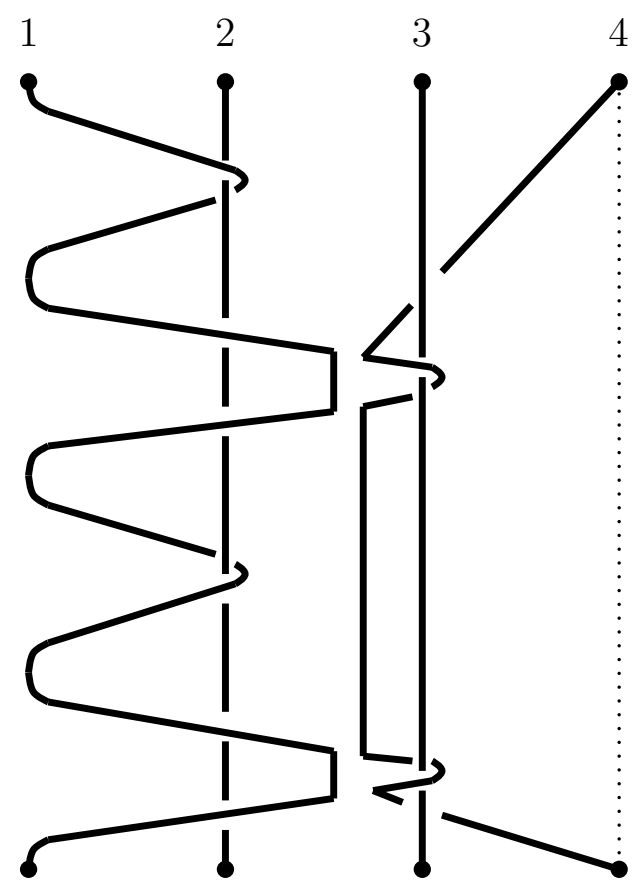

Figure 5: "merge and part" to unravel the braid of Figure 4 


\section{The merge and part braid monoid}

Let $n$ be a positive integer which is fixed throughout. Denote by $B=B_{n}$ the braid group on $n$ strings, and by $E$ the set of all equivalences on $\{1, \ldots, n\}$, which is an upper semilattice under $\vee$. Denote the identity elements of $B$ and $E$ by 1 (which has to be read in context) and the zero of $E$ by 0 .

If $\beta \in B$ then $\bar{\beta}$ denotes the associated permutation, so that overline is a group homomorphism from $B$ onto the symmetric group. If $\mathcal{E} \in E$ then put

$$
\mathcal{E}^{\beta}=\{(i, j) \mid(i, j) \bar{\beta} \in \mathcal{E}\},
$$

where we define $(i, j) \bar{\beta}=(i \bar{\beta}, j \bar{\beta})$. If follows quickly that

$$
\phi: B \rightarrow \text { Aut } E, \beta \mapsto \beta \phi: \mathcal{E} \mapsto \mathcal{E}^{\beta}
$$

is an antihomomorphism, so we get the semidirect product

$$
E \rtimes B=E \rtimes_{\phi} B=\{(\mathcal{E}, \beta) \mid \mathcal{E} \in E, \beta \in B\}
$$

with multiplication $(\mathcal{E}, \beta)\left(\mathcal{E}_{0}, \beta_{0}\right)=\left(\mathcal{E} \vee \mathcal{E}_{0}^{\beta}, \beta \beta_{0}\right)$. It is routine now to check that $E \rtimes B$ is a factorizable inverse semigroup with group of units $\{(1, \beta) \mid \beta \in B\} \cong B$ and set of idempotents $\{(\mathcal{E}, 1) \mid \mathcal{E} \in E\} \cong E$. (The definition and properties of factorizability are reviewed and developed in the next section.)

For each $i=1, \ldots, n-1$ denote by $\sigma_{i}$ the usual braid generator where the $i$ th string crosses over the $(i+1)$ th string. For each $\mathcal{E} \in E$ define the subgroup

$$
B_{\mathcal{E}}=\left\langle\beta^{-1} \sigma_{i} \beta \mid i \in\{1, \ldots, n-1\},(i, i+1) \bar{\beta} \in \mathcal{E}\right\rangle
$$

(which we interpret to be the trivial subgroup when $\mathcal{E}$ is the identity equivalence relation). The following facts are immediate from the definitions:

$$
\begin{aligned}
& B_{\mathcal{E}} \subseteq B_{\mathcal{E}^{\prime}} \quad \text { if } \quad \mathcal{E} \subseteq \mathcal{E}^{\prime}, \\
& \mathcal{E}^{\beta}=\mathcal{E} \quad \text { if } \quad \beta \in B_{\mathcal{E}},
\end{aligned}
$$




$$
\gamma B_{\mathcal{E}} \gamma^{-1}=B_{\mathcal{E} \gamma} \text { for all } \gamma \in B
$$

Define an equivalence $\sim$ on $E \rtimes B$ by

$$
(\mathcal{E}, \beta) \sim\left(\mathcal{E}_{0}, \beta_{0}\right) \quad \text { if and only if } \quad \mathcal{E}=\mathcal{E}_{0} \quad \text { and } \quad \beta \beta_{0}^{-1} \in B_{\mathcal{E}}
$$

Lemma 2.1. The equivalence $\sim i s$ a congruence.

Proof. Suppose $\left(\mathcal{E}_{1}, \beta_{1}\right) \sim\left(\mathcal{E}_{1}^{\prime}, \gamma_{1}\right)$ and $\left(\mathcal{E}_{2}, \beta_{2}\right) \sim\left(\mathcal{E}_{2}^{\prime}, \gamma_{2}\right)$. Then $\mathcal{E}_{1}=\mathcal{E}_{1}^{\prime}$, $\mathcal{E}_{2}=\mathcal{E}_{2}^{\prime}, \beta_{1} \gamma_{1}^{-1} \in B_{\mathcal{E}_{1}}$ and $\beta_{2} \gamma_{2}^{-1} \in B_{\mathcal{E}_{2}}$, so that

$$
\begin{aligned}
\beta_{1} \beta_{2}\left(\gamma_{1} \gamma_{2}\right)^{-1} & =\left(\beta_{1} \gamma_{1}^{-1}\right) \gamma_{1}\left(\beta_{2} \gamma_{2}^{-1}\right) \gamma_{1}^{-1} \\
& \in B_{\mathcal{E}_{1}} \gamma_{1} B_{\mathcal{E}_{2}} \gamma_{1}^{-1} \subseteq B_{\mathcal{E}_{1} \vee \mathcal{E}_{2}}^{\gamma_{1}}
\end{aligned}
$$

Further, since $\gamma_{1} \beta_{1}^{-1}=\left(\beta_{1} \gamma_{1}^{-1}\right)^{-1} \in B_{\mathcal{E}_{1}} \subseteq B_{\mathcal{E}_{1} \vee \mathcal{E}_{2}^{\beta_{1}}}$, and $\left(\gamma_{1} \beta_{1}^{-1}\right) \phi \in$ Aut $E$, we have

$$
\begin{aligned}
\mathcal{E}_{1} \vee \mathcal{E}_{2}^{\beta_{1}} & =\left(\mathcal{E}_{1} \vee \mathcal{E}_{2}^{\beta_{1}}\right)^{\gamma_{1} \beta_{1}^{-1}} \\
& =\mathcal{E}_{1}^{\gamma_{1} \beta_{1}^{-1}} \vee\left(\mathcal{E}_{2}^{\beta_{1}}\right)^{\gamma_{1} \beta_{1}^{-1}} \\
& =\mathcal{E}_{1} \vee \mathcal{E}_{2}^{\left(\gamma_{1} \beta_{1}^{-1} \beta_{1}\right)} \\
& =\mathcal{E}_{1} \vee \mathcal{E}_{2}^{\gamma_{1}}
\end{aligned}
$$

This proves $\left(\mathcal{E}_{1}, \beta_{1}\right)\left(\mathcal{E}_{2}, \beta_{2}\right) \sim\left(\mathcal{E}_{1}^{\prime}, \gamma_{1}\right)\left(\mathcal{E}_{2}^{\prime}, \gamma_{2}\right)$.

Define the merge and part braid monoid on $n$ strings to be

$$
\widetilde{B}=\widetilde{B}_{n}=(E \rtimes B) / \sim .
$$

Denote the $\sim$-congruence class of $(\mathcal{E}, \beta)$ by $[\mathcal{E}, \beta]$. Clearly, the natural map $(\mathcal{E}, \beta) \mapsto[\mathcal{E}, \beta]$ is one-one on $\{(1, \beta) \mid \beta \in B\}$ and on $\{(\mathcal{E}, 1) \mid \mathcal{E} \in E\}$, so we get the following:

Proposition 2.2. The inverse monoid $\widetilde{B}$ is factorizable with group of units $\{[1, \beta] \mid \beta \in B\} \cong B$ and semilattice of idempotents $\{[\mathcal{E}, 1] \mid \mathcal{E} \in E\} \cong E$. 
We now provide a geometric realization of $\widetilde{B}$ which justifies the manipulations of strings used in the examples in the first section. Let

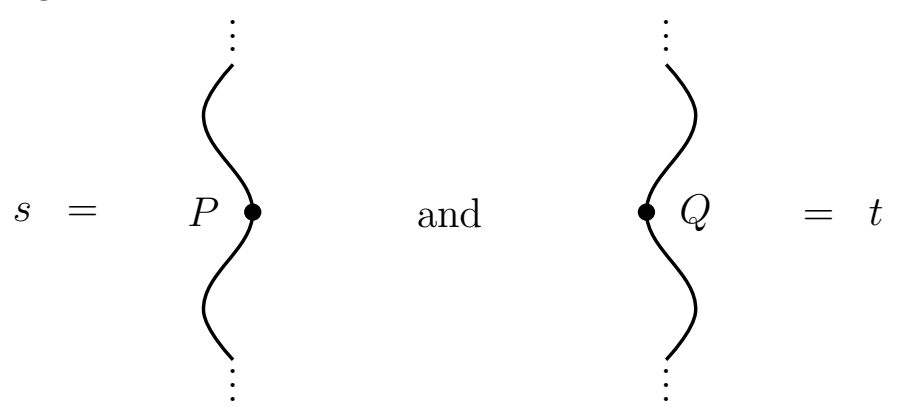

be two strings descending from fixed points on the upper plane to connect to fixed points on the lower plane. We say that a homotopy causes $s$ and $t$ to merge and part if, during the homotopy, $s$ and $t$ come together just once at say $P$ and $Q$,

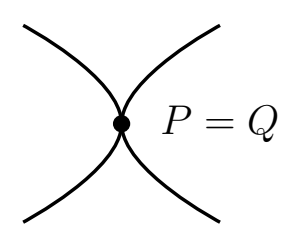

and then part, reconstituting as two strings made up of respective upper and lower strands. A catalogue of the possible configurations in the neighbourhood a moment before and after is given in Figure 6. Note that (2) and (3) can be interchanged using normal homotopy. Also an interchange between (6) and (7) can be achieved using two interchanges between types (4) and (5) in nearby neighbourhoods.

Consider a configuration of strings $s_{1}, \ldots, s_{n}$ emanating from points $1, \ldots, n$ respectively on the upper plane, and let $\mathcal{E} \in E$. We say $s_{i}$ is $\mathcal{E}$-equivalent to $s_{j}$ if $(i, j) \in \mathcal{E}$, and $\mathcal{E}$-inequivalent to $s_{j}$ if $(i, j) \notin \mathcal{E}$.

Theorem 2.3. Let $\mathcal{E}, \mathcal{E}_{0} \in E$ and $\beta, \beta_{0} \in B$. Then $(\mathcal{E}, \beta) \sim\left(\mathcal{E}_{0}, \beta_{0}\right)$ if and only if $\mathcal{E}=\mathcal{E}_{0}$ and there exists a homotopy from a representative of $\beta$ to a representative of $\beta_{0}$ such that, in the course of the homotopy, $\mathcal{E}$-inequivalent strings never touch and $\mathcal{E}$-equivalent string are allowed to merge and part (one at a time, a finite number of times). 


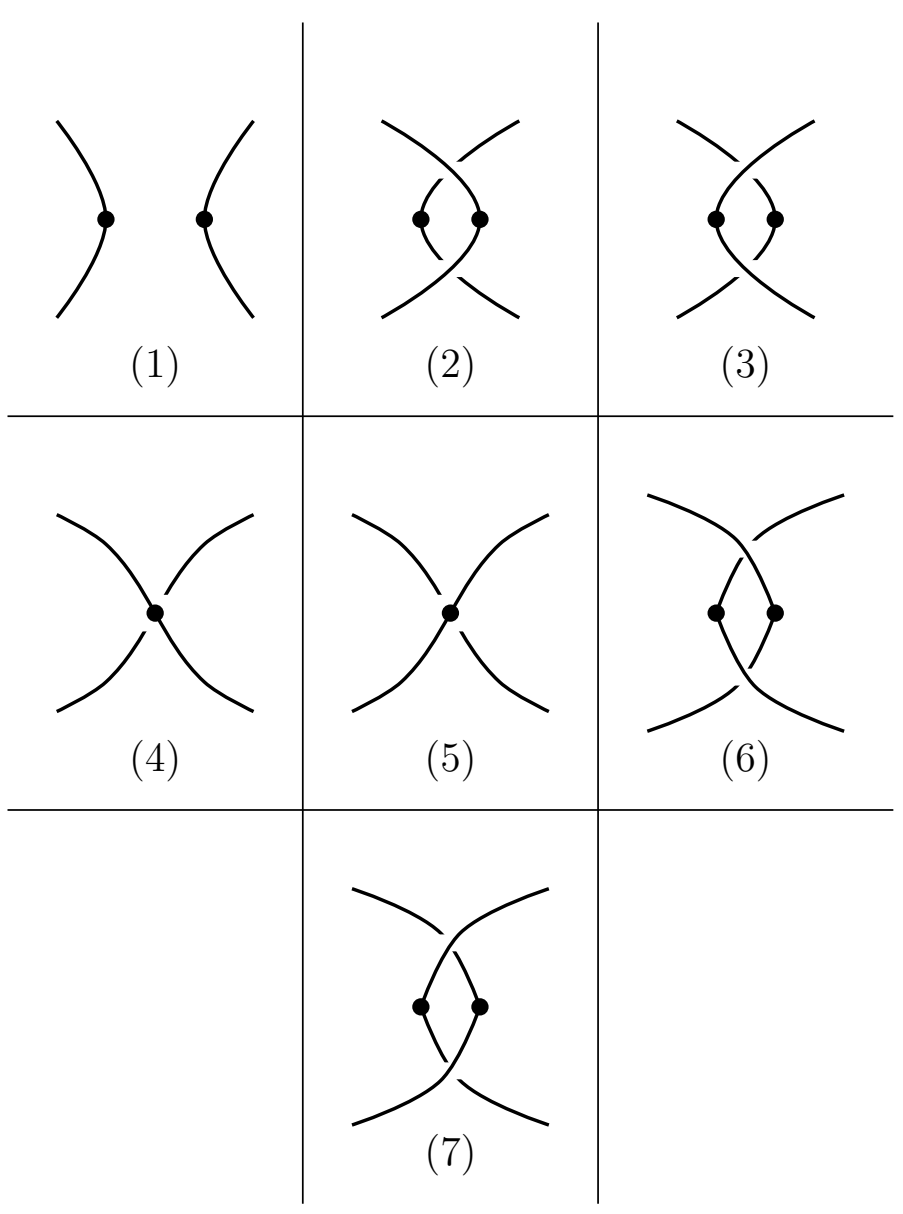

Figure 6: possibilities before and after "merge and part"

Proof. $(\Rightarrow)$ Suppose that $(\mathcal{E}, \beta) \sim\left(\mathcal{E}_{0}, \beta_{0}\right)$. Then $\mathcal{E}=\mathcal{E}_{0}$ and $\beta \beta_{0}^{-1} \in B_{\mathcal{E}}$. We prove there is a homotopy of the required type from a representative of $\beta$ to a representative of $\beta_{0}$ by induction on the number of generators of $B_{\mathcal{E}}$ in the product forming $\beta \beta_{0}^{-1}$. If no generators are required then $\beta \beta_{0}^{-1}=1$ so there is a homotopy in which no strings touch, which starts an induction. Thus, to prove the inductive step, it is sufficient to check that there is a homotopy of the required type between representatives of 1 and $\gamma^{-1} \sigma_{i} \gamma$ where $i \in\{1, \ldots, n-1\}$ and $(i, i+1) \bar{\gamma} \in \mathcal{E}$. Put

$$
j=i \bar{\gamma}, \quad k=(i+1) \bar{\gamma} .
$$

Let $\widehat{\gamma}$ be a representative of $\gamma$ and $\hat{1}$ the straight string representative of the identity braid 1 . We get a homotopy from $\widehat{1}$ to a representative of $\gamma^{-1} \sigma_{i} \gamma$ as the composite of $H_{1}$ and $H_{2}$ displayed in Figure 7 (where $\widehat{\gamma}^{-1}$ is the reflection of $\widehat{\gamma}$ in a horizontal plane, and the representatives have been contracted in the second and third parts of the diagram). It is to be understood in Figure 7 that $H_{1}$ is a homotopy where no strings touch and $H_{2}$ is a homotopy in 


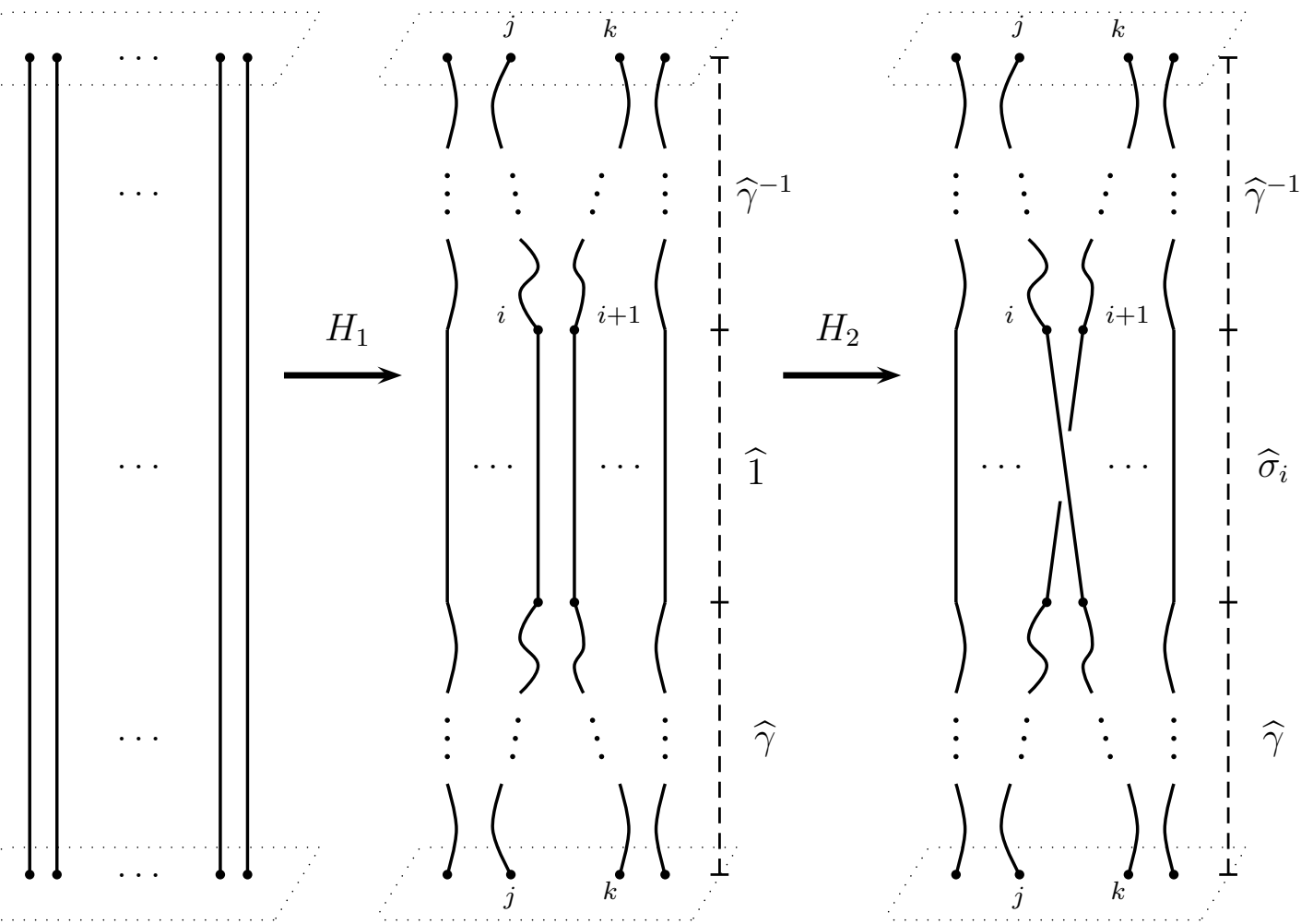

Figure 7: "merge and part" homotopy from $\hat{1}$ to a representative of $\gamma^{-1} \sigma_{i} \gamma$

which only the $j$ th and $k$ th strings have merged and parted.

$(\Leftarrow)$ Suppose now $\mathcal{E}=\mathcal{E}_{0}$ and a homotopy $H$ exists of the required type between a representative $\widehat{\beta}$ of $\beta$ and a representative $\widehat{\beta_{0}}$ of $\beta_{0}$. If no strings touch then $\beta=\beta_{0}$, so certainly $\beta \beta_{0}^{-1} \in B_{\mathcal{E}}$, which starts an induction. Suppose $H$ is the composite of homotopies $H_{1}$ and $H_{2}$ where during $H_{2}$ exactly one pair of strings merge and part. Let $\gamma$ be the braid of the representative $\widehat{\gamma}$ resulting from applying $H_{1}$ to $\widehat{\beta}$. By an inductive hypothesis

$$
\beta \gamma^{-1} \in B_{\mathcal{E}}
$$

The homotopy $H_{2}$ can be replaced (if necessary) by a composite $H_{3} H_{4} H_{5}$ where $\gamma=\gamma_{1} \gamma_{2}$ for some $\gamma_{1}, \gamma_{2} \in B$,

$$
\widehat{\gamma} \stackrel{H_{3}}{\longrightarrow}\left|\begin{array}{c}
\widehat{\gamma}_{1} \\
\widehat{1} \\
\widehat{\gamma}_{2}
\end{array}\right| \stackrel{H_{4}}{\longrightarrow}\left|\begin{array}{c}
\widehat{\gamma}_{1} \\
\widehat{\tau} \\
\widehat{\gamma}_{2}
\end{array}\right| \stackrel{H_{5}}{\longrightarrow} \widehat{\beta}_{0}
$$

and $\tau \in\left\{1, \sigma_{i}, \sigma_{i}^{-1}, \sigma_{i}^{2}, \sigma_{i}^{-2}\right\}$ (according to cases (1) to (7) catalogued in 
Figure 6 ), where $H_{3}$ and $H_{5}$ have no strings touching, and $H_{4}$ alters the $\widehat{1}$, contracted in the middle, by causing the $i$ th and $(i+1)$ th strings to merge and part. Because no strings touch during $H_{5}$ we have

$$
\beta_{0}=\gamma_{1} \tau \gamma_{2}
$$

In order for $H_{4}$ to apply to the $i$ th and $(i+1)$ the strings, we need $(i, i+1) \bar{\gamma}_{1}^{-1} \in$ $\mathcal{E}$. Thus $\gamma_{1} \tau \gamma_{1}^{-1} \in B_{\mathcal{E}}$, so

$$
\beta \beta_{0}^{-1}=\beta \gamma_{2}^{-1} \tau^{-1} \gamma_{1}^{-1}=\beta \gamma^{-1} \gamma_{1} \tau^{-1} \gamma_{1}^{-1} \in B_{\mathcal{E}},
$$

and the theorem is proved.

In the final section of this paper we will return to discuss this monoid and prove it embeds in the coset monoid of the braid group.

\section{Coset monoids of groups and duality}

In this section our aim is to use coset monoids of groups to dualize the following result of Munn [8]:

Theorem 3.1. If $S$ is an inverse semigroup with semilattice of idempotents $E$ then there exists a homomorphism from $S$ into $T_{E}$ with kernel $\mu$, the largest congruence contained in $\mathcal{H}$. Thus, if $S$ is fundamental, then $S$ embeds in $T_{E}$. Moreover every full inverse subsemigroup of $T_{E}$ is fundamental.

Coset monoids of groups were first studied by Schein [10], who discusses them in detail, and with generalizations to semigroups, in [11]. Other authors, such as McAlister [7], Leech [6], Nambooripad and Veeramony [9], have used them and generalizations in various contexts.

We will introduce the topic using the following simple example. Let $G$ denote the symmetric group on a set of size 3 , which has the presentation $\left\langle a, b \mid a^{3}=b^{2}=1, a^{b}=a^{-1}\right\rangle$. If we identify $G$ also with the group of symmetries of the triangle then we may list the elements of $G$ as $\left\{1, a, a^{2}, b, a b, a^{2} b\right\}$, 
where $1, a, a^{2}$ form a normal subgroup of rotations and each of $b, a b, a^{2} b$ is a reflection generating a nonnormal subgroup of order 2 . Let $\mathcal{S}$ denote the lattice of subgroups of $G$. In particular $\mathcal{S}$ is a semilattice with respect to $\vee$ defined by $H \vee K=\langle H \cup K\rangle$ whose Hasse diagram may be depicted thus:

\section{$\langle 1\rangle$}

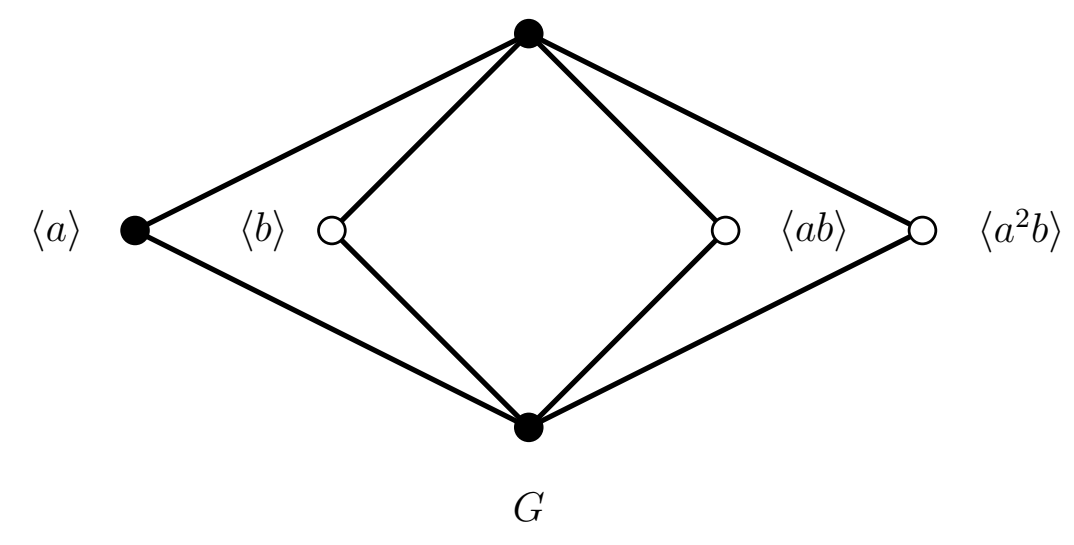

- = normal

$\mathrm{O}=$ nonnormal

Then $G$ acts on $\mathcal{S}$ by conjugation, fixing the normal subgroups and providing permutations of the nonnormal subgroups. (In this example the nonnormal subgroups provide a faithful permutation representation.) We may form

$$
T=\mathcal{S} \rtimes G=\{(H, g) \mid H \leq G, g \in G\}
$$

with multiplication $(H, g)(K, \ell)=\left(H \vee K^{g}, g \ell\right)$. It is not hard to see (Figure 8) that the $\mathcal{D}$-classes of $T$ correspond to conjugacy classes of subgroups of $G$, and that, in our example, the nonnormal subgroups lie in a single $\mathcal{D}$-class. Define a congruence $\sim$ on $T$ by

$$
(H, g) \sim(K, \ell) \text { if and only if } H g=K \ell,
$$

equality of cosets. As before, write $[H, g]$ for the congruence class of $(H, g)$, so

$$
T / \sim=\{[H, g] \mid H \leq G, g \in G\}
$$




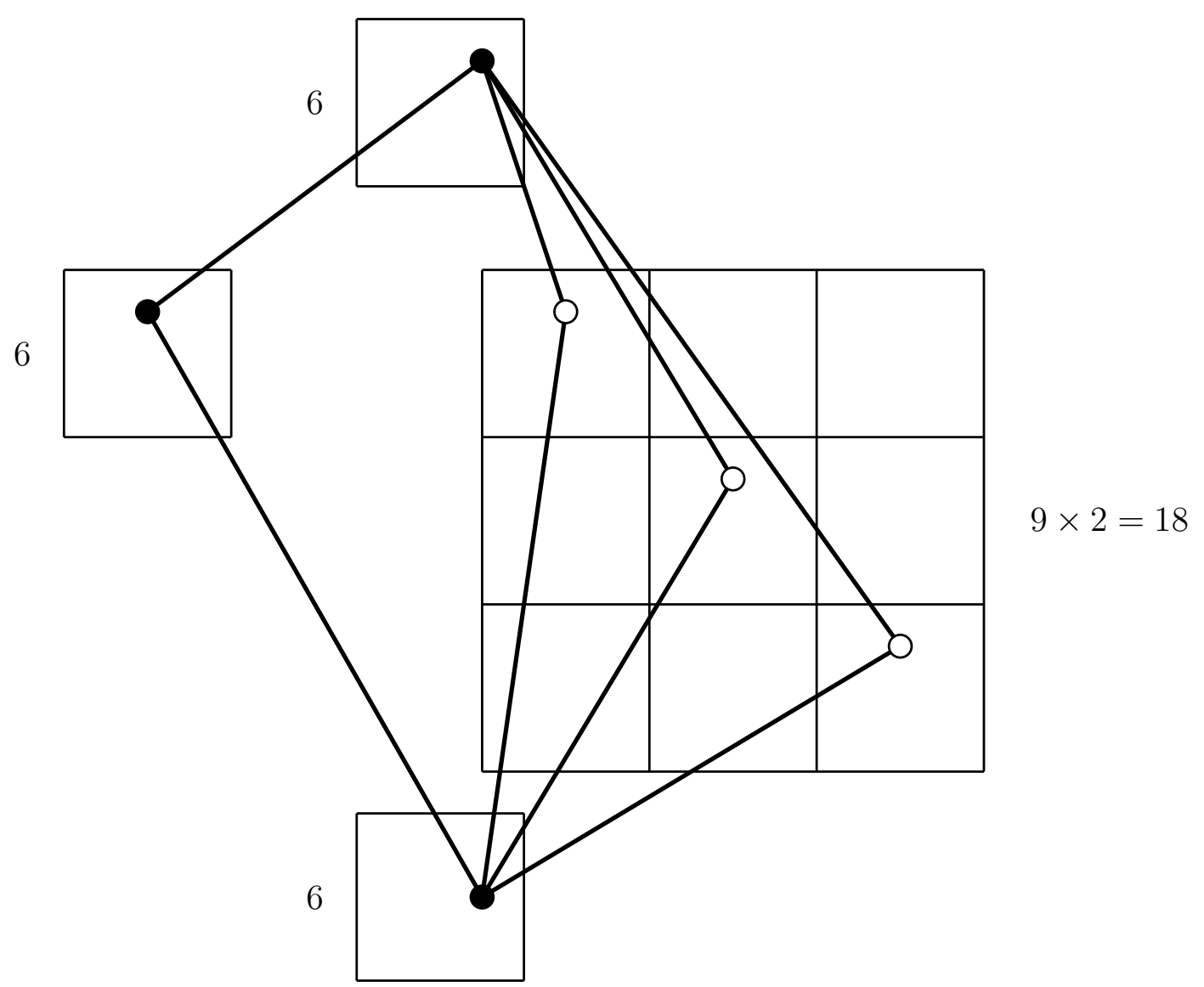

Figure 8: eggbox diagram for $T=\mathcal{S} \rtimes G$ containing 36 elements

with multiplication $[H, g][K, \ell]=\left[H \vee K^{g}, g \ell\right]$. But there is a bijection between $T / \sim$ and

$$
\mathcal{C}(G)=\{H g \mid H \leq G, g \in G\}
$$

the set of all cosets with respect to all subgroups of $G$. Thus $\mathcal{C}(G)$ inherits the multiplication

$$
(H g) *(K \ell)=\left(H \vee K^{g}\right) g \ell,
$$

which one may show is the smallest coset containing the set product $\mathrm{HgK \ell}$. We call $(\mathcal{C}(G), *)$ the coset monoid of $G$. (It is denoted by $K(G)$ by Schein and others, but we prefer our present notation because of a certain universal property with respect to a class $\mathcal{C}$ defined below.) In our example $\mathcal{C}(G) \cong$ $T / \sim$ has 18 elements, displayed in Figure 9.

All of the preceding definitions of this section are now taken as read for any group $G$. Recall that an inverse monoid $M$ is factorizable if $M=G E(=E G)$ where $G$ is its group of units and $E$ is its semilattice of idempotents, and it 


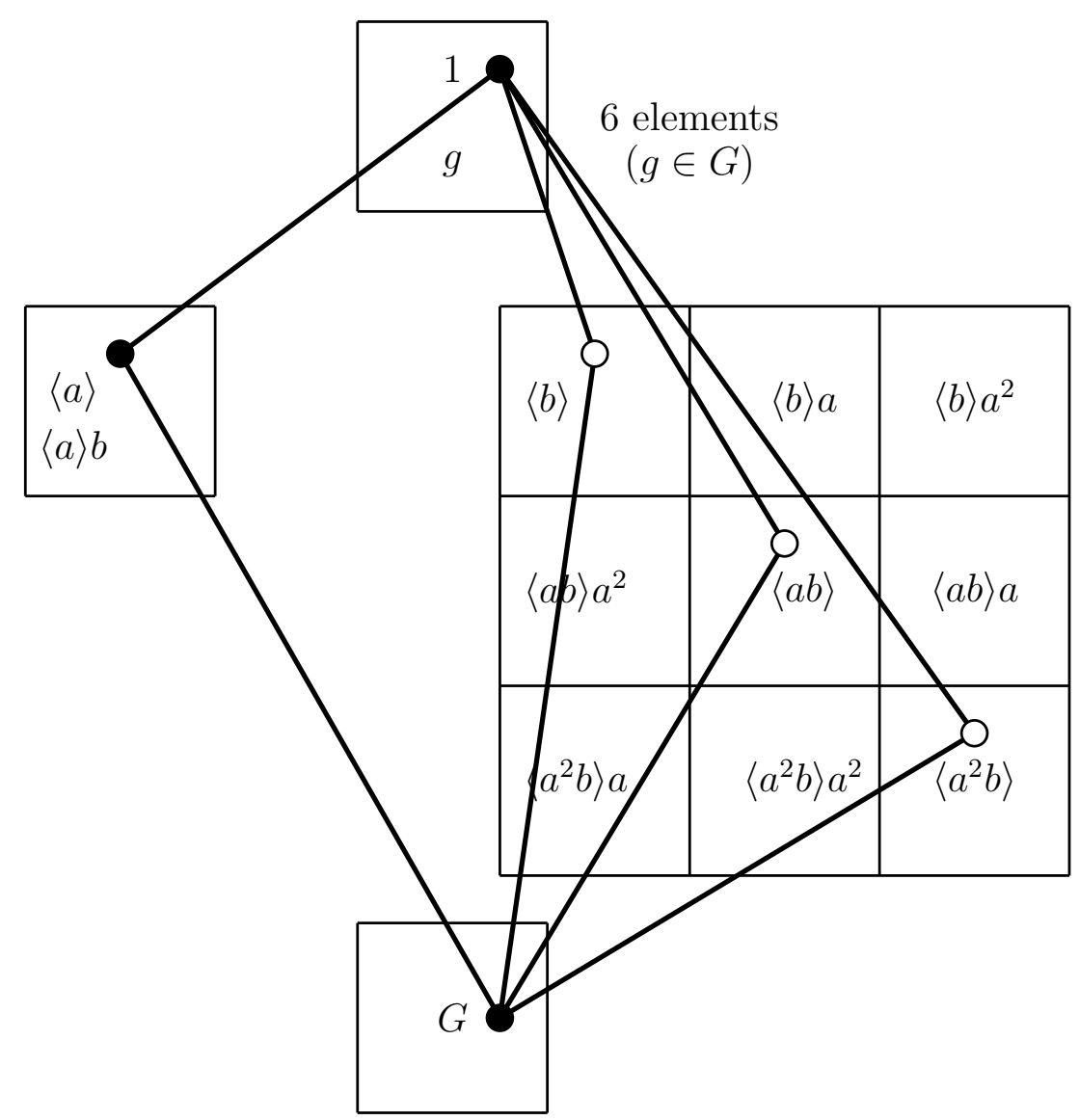

Figure 9: eggbox diagram for $\mathcal{C}(G)$ containing 18 elements

is standard to write $G_{e}$ for the stabilizer of $e \in E$, that is,

$$
G_{e}=\{g \in G \mid g e=e\}=\{g \in G \mid e g=e\} .
$$

(The reader may easily check that, in the previous section, where $B$ is the braid group and $\mathcal{E}$ is an equivalence on $\{1, \ldots, n\}$, the definition of $B_{\mathcal{E}}$ there gives precisely the definition of $B_{\mathcal{E}}$ here as a stabilizer.) Note that, for $g, h \in$ $G$ and $e, f \in E$, using cancellation by a unit,

$$
g e=f \quad \Longrightarrow \quad g e g e=g e, e=e g e=e f=f e=f
$$

and dually

$$
e g=f \quad \Longrightarrow \quad e=f,
$$

from which it follows quickly that

$$
e g \mathcal{R} f h \quad \Longleftrightarrow \quad e=f \quad \Longleftrightarrow \quad g e \mathcal{L} f h .
$$

Now define $\mathcal{C}$ to be the class of factorizable inverse monoids $M=G E$ such that the mapping

$$
e \mapsto G_{e}: E \rightarrow \mathcal{S}
$$


respects joins, that is, $G_{e f}=\left\langle G_{e} \cup G_{f}\right\rangle$ for all $e, f \in E$.

Clearly $\mathcal{C}(G)$ is factorizable with $G$ as its group of units, and if $H, K \leq G$ then $G_{H}=H$, so that

$$
G_{H * K}=G_{\langle H \cup K\rangle}=\langle H \cup K\rangle .
$$

This verifies that

$$
\mathcal{C}(G) \in \mathcal{C}
$$

Clearly also, since all stabilizers are trivial, $\mathcal{S} \rtimes G \in \mathcal{C}$. In fact, $\mathcal{C}(G)$ is a cofundamental image of $\mathcal{S} \rtimes G$ in the sense of Theorem 3.5 below, which dualizes Theorem 3.1.

Lemma 3.2. Let $M=G E \in \mathcal{C}$ and $\rho$ be a congruence on $M$. Then

$$
\rho \cap \mathcal{R}=1 \quad \Longleftrightarrow \quad \rho \cap \mathcal{L}=1 \text {. }
$$

Proof. ( $\Longrightarrow$ Suppose $\rho \cap \mathcal{R}=1$. Then, for all $g, h \in G, e, f \in E$,

$$
\begin{aligned}
(g e, h f) \in \rho \cap \mathcal{L} & \Longrightarrow\left(e g^{-1}, f h^{-1}\right) \in \rho \cap \mathcal{R} \\
& \Longrightarrow e g^{-1}=f h^{-1} \\
& \Longrightarrow g e=\left(e g^{-1}\right)^{-1}=\left(f h^{-1}\right)^{-1}=h f .
\end{aligned}
$$

This proves $\rho \cap \mathcal{L}=1$. The proof of $(\Longleftarrow)$ is similar.

Lemma 3.3. Let $M=G E \in \mathcal{C}$. Then

$$
\theta: M \rightarrow \mathcal{C}(G), e g \mapsto G_{e} g\left[g e \mapsto g G_{e}\right],
$$

is a homomorphism.

Proof. Let $g, h \in G$ and $e, f \in E$. Note $\theta$ is well-defined because if $e g=f h$ then $e=f$ and $e g h^{-1}=e$, so $g h^{-1} \in G_{e}$, giving $G_{e} g=G_{e} h=G_{f} h$. Further, by definition of membership of $\mathcal{C}$,

$$
\begin{aligned}
(e g f h) \theta & =\left(e g f g^{-1} g h\right) \theta=G_{e g f g^{-1}} g h \\
& =\left(G_{e} \vee G_{g f g^{-1}}\right) g h=\left\langle G_{e} \cup G_{g f g^{-1}}\right\rangle g h \\
& =G_{e} g * G_{f} h=(e g) \theta *(f h) \theta .
\end{aligned}
$$


Lemma 3.4. Let $M=G E \in \mathcal{C}$. Then there exists a largest congruence $\nu$ on $M$ such that $\nu \cap \mathcal{R}=1$ or (equivalently) $\nu \cap \mathcal{L}=1$. Further there is a representation of $M$ by $\mathcal{C}(G)$,

$$
\theta: M \rightarrow \mathcal{C}(G), e g \mapsto G_{e} g\left[g e \mapsto g G_{e}\right]
$$

whose kernel is $\nu$.

Proof. By the previous lemma, $\theta$ is a representation. Clearly

$$
\operatorname{ker} \theta=\left\{(e g, f h) \mid G_{e}=G_{f} \quad \text { and } \quad g h^{-1} \in G_{e}\right\} \text {. }
$$

Further,

$$
(e g, f h) \in \operatorname{ker} \theta \cap \mathcal{R} \quad \Longrightarrow \quad e=f \text { and } g h^{-1} \in G_{e} \quad \Longrightarrow \quad e g=e h
$$

which proves $\operatorname{ker} \theta \cap \mathcal{R}=1$.

Let $\rho$ be any congruence on $M$ such that $\rho \cap \mathcal{R}=1$. To complete the proof it suffices to show $\rho \subseteq \operatorname{ker} \theta$. Suppose $(e g, f h) \in \rho$. Then $\left(g^{-1} e, h^{-1} f\right) \in \rho$ so

$$
e=e g g^{-1} \text { e } \rho f h h^{-1} f \rho f \text {. }
$$

If $g \in G_{e}$ then

$$
f \rho e=e g \rho f g
$$

so $f=f g$, since $f \mathcal{R} f g$ and $\rho \cap \mathcal{R}=1$, giving $g \in G_{f}$. Thus $G_{e} \subseteq G_{f}$ and similarly $G_{f} \subseteq G_{e}$ whence equality holds. But also

$$
\text { eg } \rho \text { fh } \rho \text { eh and eg } \mathcal{R} \text { eh }
$$

so $e g=e h$, whence $g h^{-1} \in G_{e}$. This proves $(e g, f h) \in \operatorname{ker} \theta$, so $\rho \subseteq \operatorname{ker} \theta$.

Call $M \in \mathcal{C}$ cofundamental if $\nu=1$, and call an inverse submonoid $N$ of $M$ cofull if $N \in \mathcal{C}$ and $N$ has the same group of units as $M$.

Theorem 3.5. If $M=G E \in \mathcal{C}$ then

$$
\theta: M \rightarrow \mathcal{C}(G), e g \mapsto G_{e} g\left[g e \mapsto g G_{e}\right]
$$


is a representation with kernel $\nu$ equal to the largest congruence on $M$ such that

$$
\nu \cap \mathcal{R}=1_{M}
$$

Thus if $M$ is cofundamental then $M$ embeds in $\mathcal{C}(G)$ as a cofull inverse submonoid. Moreover every cofull inverse submonoid of $\mathcal{C}(G)$ is cofundamental.

Proof. In light of the previous lemmas, it remains to prove the final statement. Let $M$ be a cofull inverse submonoid of $\mathcal{C}(G)$. If $(H g, K \ell) \in \nu$ then

$$
H=G_{H}=G_{K}=K \text { and } g \ell^{-1} \in G_{H}=H
$$

so $H g=K \ell$. Thus $\nu=1$ and $M$ is cofundamental.

The duality between Theorems 3.1 and 3.5 is perhaps unexpected. Observe that a congruence $\rho$ on an inverse monoid $M$ is idempotent-separating if and only if

$$
\rho \subseteq \mathcal{H}=\mathcal{R} \cap \mathcal{L}
$$

that is,

$$
\rho \subseteq \mathcal{R} \quad \text { and } \quad \rho \subseteq \mathcal{L} .
$$

One's first guess at a dual property for $\rho$ might be be to make it "antipodal" to $\mathcal{H}$ :

$$
\rho \cap \mathcal{H}=1_{M}
$$

But this won't lead to a result like Theorem 3.5, because of the example $M$ diplayed in Figure 10, which is a cofull submonoid of $\mathcal{C}(G)$ (displayed in Figure 9) where $G$ is the symmetric group on three letters. Observe that $\mathcal{H}$ is trivial on the ideal $M \backslash G$, and the Rees congruence $\rho$ with respect to this ideal certainly is not trivial, yet $\rho \cap \mathcal{H}=1_{M}$.

One's second guess at a dual property for $\rho$ might be be to make it "antipodal" to the "dual" of $\mathcal{H}$, which one might think of as $\mathcal{D}$ :

$$
\rho \cap \mathcal{D}=1_{M} .
$$




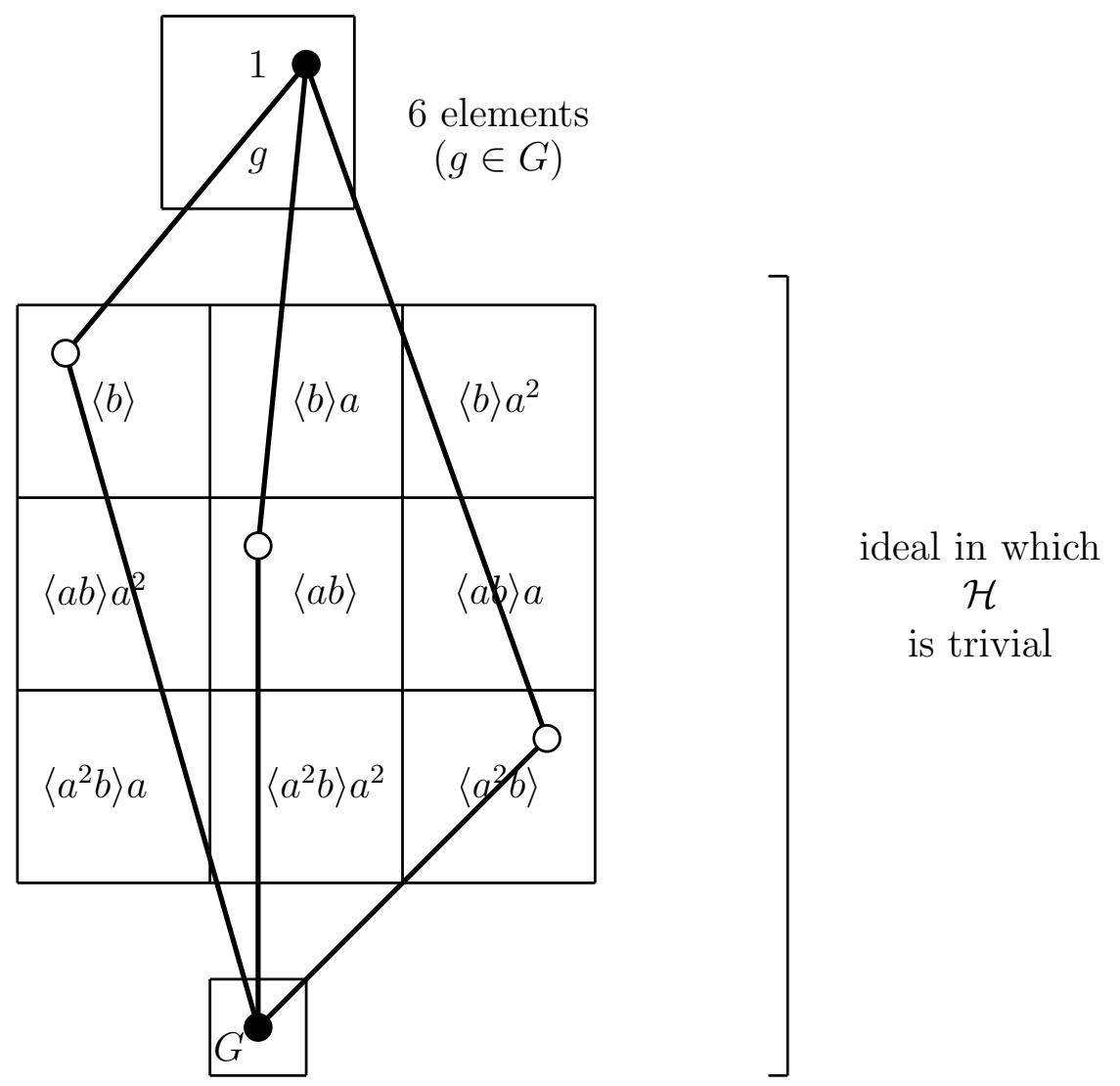

Figure 10: eggbox diagram for a cofull submonoid of $\mathcal{C}(G)$ where $G$ is the symmetric group on 3 letters

But then this won't lead to a result like Theorem 3.5 either, because for any group $G$ with lattice of subgroups $\mathcal{S}$, the representation $\theta: \mathcal{S} \rtimes G \rightarrow$ $\mathcal{C}(G)$ becomes projection onto the second coordinate $(e, g) \mapsto g$ (since all the stabilizers are trivial), and certainly in nontrivial examples, $\operatorname{ker} \theta \cap \mathcal{D} \neq 1_{\mathcal{S} \rtimes G}$.

The correct property turns out to first take the logical dual of the conjunction $\rho \subseteq \mathcal{R}$ and $\rho \subseteq \mathcal{L}$, which is a disjunction, and then make $\rho$ "antipodal" to each alternative:

$$
\rho \cap \mathcal{R}=1_{M} \quad \text { or } \quad \rho \cap \mathcal{L}=1_{M} .
$$

But because of the equivalence of these two alternatives (Lemma 3.2) only one needs to be included in the statement of Theorem 3.5. 


\section{An embedding in the coset monoid of the braid group}

In this final section we prove that the merge and part braid monoid embeds in the coset monoid of the braid group. Let $X=\{1, \ldots, n\}$ where $n$ is a fixed positive integer, and let $E$ be the set of equivalence relations on $X$. Recall $B=B_{n}$ denotes the braid group on $n$ strings and $\widetilde{B}=(E \rtimes B) / \sim$ is the merge and part monoid defined in the second section. The key step is the following:

Lemma 4.1. Let $\mathcal{E}_{1}, \mathcal{E}_{2} \in E$. Then $B_{\mathcal{E}_{1}} \vee B_{\mathcal{E}_{2}}=B_{\mathcal{E}_{1} \vee \mathcal{E}_{2}}$.

Proof. The forward set containment is obvious. Equality is also obvious if $\mathcal{E}_{1}$ and $\mathcal{E}_{2}$ are both the identity equivalence. Suppose then that at least one of these is not the identity equivalence. Let $\tau=\beta^{-1} \sigma_{i} \beta$ be a generator of $B_{\mathcal{E}_{1} \vee \mathcal{E}_{2}}$ where $i \in X \backslash\{n\}$ and $\beta \in B$ such that

$$
(i, i+1) \bar{\beta} \in \mathcal{E}_{1} \vee \mathcal{E}_{2}
$$

To complete the proof of the lemma, it suffices to show $\tau \in B_{\mathcal{E}_{1}} \vee B_{\mathcal{E}_{2}}$. Since $\mathcal{E}_{1} \vee \mathcal{E}_{2}$ is the transitive closure of $\mathcal{E}_{1} \cup \mathcal{E}_{2}$, without loss of generality we may suppose there exists a positive integer $m$ and $x_{1}, \ldots, x_{2 m} \in X$ such that

$$
i \bar{\beta}=x_{1} \mathcal{E}_{1} x_{2} \mathcal{E}_{2} \ldots \mathcal{E}_{1} x_{2 m-2} \mathcal{E}_{2} x_{2 m-1} \mathcal{E}_{1} x_{2 m}=(i+1) \bar{\beta}
$$

For $a, b \in X, a<b$, put

$$
\delta_{a, b}=\sigma_{a+1} \sigma_{a+2} \ldots \sigma_{b-1}
$$

(interpreted as the identity braid if $b=a+1$ ) and

$$
\gamma_{a, b}=\gamma_{b, a}=\delta_{a, b}^{-1} \sigma_{a} \delta_{a, b}
$$

so that $\overline{\gamma_{a, b}}$ is the transposition interchanging $a$ and $b$ and

$$
(a, a+1) \overline{\delta_{a, b}}=(a, b) .
$$


Thus, by definition, $\gamma_{c, d}$ is a generator of $B_{\mathcal{E}}$ for all $\mathcal{E} \in E$ and $(c, d) \in \mathcal{E}$ with $c \neq d$. Now put

$$
\delta=\gamma_{x_{1}, x_{2}} \gamma_{x_{2}, x_{3}} \cdots \gamma_{x_{2 m-2}, x_{2 m-1}}
$$

(interpreted as the identity braid if $m=1$ ) and

$$
\gamma=\delta \gamma_{x_{2 m-1}, x_{2 m}} \delta^{-1}
$$

Observe that

$$
\gamma_{x_{i}, x_{i+1}} \in \begin{cases}B_{\mathcal{E}_{1}} & \text { if } i \text { is odd } \\ B_{\mathcal{E}_{2}} & \text { if } i \text { is even }\end{cases}
$$

so that $\gamma \in\left\langle B_{\mathcal{E}_{1}} \cup B_{\mathcal{E}_{2}}\right\rangle$. Also

$$
(i, i+1) \overline{\beta \gamma}=\left(x_{1}, x_{2 m}\right) \bar{\gamma}=\left(x_{2}, x_{1}\right)
$$

so that $(\beta \gamma)^{-1} \sigma_{i} \beta \gamma \in B_{\mathcal{E}_{1}}$. Hence

$$
\begin{aligned}
\tau & =\gamma\left(\gamma^{-1} \beta^{-1} \sigma_{i} \beta \gamma\right) \gamma^{-1} \\
& \in\left\langle B_{\mathcal{E}_{1}} \cup B_{\mathcal{E}_{2}}\right\rangle B_{\mathcal{E}_{1}}\left\langle B_{\mathcal{E}_{1}} \cup B_{\mathcal{E}_{2}}\right\rangle \\
& \subseteq B_{\mathcal{E}_{1}} \vee B_{\mathcal{E}_{2}} .
\end{aligned}
$$

Lemma 4.2. Let $\mathcal{E}_{1}, \mathcal{E}_{2} \in E$ such that $\mathcal{E}_{1} \neq \mathcal{E}_{2}$. Then $B_{\mathcal{E}_{1}} \neq B_{\mathcal{E}_{2}}$.

Proof. Without loss of generality we have $x, y \in X$ such that $(x, y) \in \mathcal{E}_{1}$ but $(x, y) \notin \mathcal{E}_{2}$. Then, using the notation introduced in the proof of the previous lemma, $\gamma_{x, y} \in B_{\mathcal{E}_{1}}$ and $\overline{\gamma_{x, y}}$ is the transposition interchanging $x$ and $y$. But, by a simple induction on the number of generators, if $\beta \in B_{\mathcal{E}_{2}}$ then $(x, x \bar{\beta}) \in \mathcal{E}_{2}$. Since $\left(x, x \overline{\gamma_{x, y}}\right)=(x, y) \notin \mathcal{E}_{2}$, we have that $\gamma_{x, y} \notin B_{\mathcal{E}_{2}}$, proving $B_{\mathcal{E}_{1}} \neq B_{\mathcal{E}_{2}}$.

Theorem 4.3. The merge and part monoid embeds in the coset monoid of the braid group. 
Proof. By Lemma 4.1, $\widetilde{B} \in \mathcal{C}$ so, by Theorem 3.5, $\theta: \widetilde{B} \rightarrow \mathcal{C}(B)$ is a representation. It follows quickly, by Lemma 4.2 , that $\theta$ is faithful.

The proofs in this section hold also for permutations (by overlining all braids), so we get the following result:

Corollary 4.4. The largest factorizable inverse submonoid of the dual symmetric inverse monoid on a finite set embeds in the coset monoid of the symmetric group.

Corollary 4.5. The merge and part braid monoid and the largest factorizable inverse submonoid of the dual symmetric inverse monoid on a finite set are cofundamental.

\section{References}

[1] J. Baez, "Link invariants of finite type and perturbation theory," Lett. Math. Phys. 26 (1992), 43-51.

[2] Joan S. Birman, "New points of view in knot theory," Bull. Amer. Math. Soc., 28 (1993), 253-287.

[3] D. Easdown and T.G. Lavers, "The inverse braid monoid," Advances in Mathematics, to appear.

[4] James East, "The factorisable braid monoid," in preparation.

[5] D.G. FitzGerald and Jonathan Leech, "Dual symmetric inverse monoids and representation theory," J. Austral. Math. Soc., 64 (1998), 345-367.

[6] Jonathan Leech, "Inverse monoids with a natural semilattice ordering," it Proc. London Math. Soc. 70 (1995), 146-182.

[7] D.B. McAlister, "Embedding inverse semigroups in coset semigroups," Semigroup Forum 20 (1980), 255-267. 
[8] W.D. Munn, "Uniform semilattices and bisimple inverse semigroups," Quart. J. Math. Oxford (2) 17 (1966), 151-159.

[9] K.S.S. Nambooripad and R. Veeramony, "Subdirect products of regular semigroups," Semigroup Forum 27 (1983), 265-307.

[10] B.M. Schein, "Semigroups of strong subsets," Volzhsky Matematichesky Sbornik 4 (1966), 180-186.

[11] B.M. Schein, "Cosets in groups and semigroups," Semigroups with applications, eds. Howie, Munn, Weinert, World Scientific, Singapore 1992, 205-221.

School of Mathematics and Statistics

University of Sydney, NSW 2006, Australia

de@maths.usyd.edu.au

jamese@maths.usyd.edu.au

School of Mathematics and Physics

University of Tasmania

GPO Box 252-37, Hobart, TAS 7001, Australia

D.FitzGerald@utas.edu.au 